\title{
EFEITO DA SÍLICA ATIVA NAS PROPRIEDADES DO CONCRETO LEVE COM ARGILA EXPANDIDA
}

\author{
EFFECT OF SILICA FUME IN THE PROPERTIES OF LIGHTWEIGHT CONCRETE WITH \\ EXPANDED CLAY
}

\author{
MARINA COSTA DE SOUZA | UNIEURO
}

AMÁLIA DE SOUSA DAVI | UNIEURO

YURI SOTERO BOMFIM FRAGA, MSC | UNIEURO

\begin{abstract}
RESUMO
A utilização de concreto leve $(C L)$ representa grande desafio devido a redução das propriedades mecânicas causadas pela substituição do agregado convencional por agregado leve. Frente ao exposto, este trabalho objetivou verificar o efeito da adição de $10 \%$ de sílica ativa (SA) em traços de concretos com substituição de $50 \%$ e $100 \%$ do volume de brita por argila expandida (AE). No estado fresco, foi analisado o teor de aditivo superplastificante necessário para obter a consistência de $100 \pm 20 \mathrm{~mm}$ através do ensaio de slump test. No estado endurecido, foram analisadas as resistências à tração e à compressão aos 3, 7 e 21 dias de hidratação. Foi observada diminuição do desempenho mecânico do concreto quando a brita foi substituída por AE. Apesar dessa diminuição, os concretos leves resultaram em maiores relações resistência/peso. Foi observado que a adição de SA melhorou significativamente as propriedades mecânicas dos concretos leves. Dessa forma é possível concluir que a adição de SA pode compensar a perda de desempenho mecânico provocado pela substituição do agregado graúdo convencional pela argila expandida, sem ser necessário aumentar a quantidade de cimento da mistura.
\end{abstract}

PALAVRAS-CHAVE: Concreto leve; Sílica ativa; Argila expandida; Fator de eficiência.

\begin{abstract}
The use of lightweight concrete $(L C)$ represents a great challenge due to the reduction of the mechanical properties caused by the substitution of conventional aggregate for light aggregate. In view of the above, this paper aimed to verify the effect of the addition of $10 \%$ of silica fume (SF) in mixtures of concrete with replacement of $50 \%$ and $100 \%$ of the volume of crushed stone by expanded clay (EC). In the fresh state, the content of superplasticizer additive needed to obtain a consistency of $100 \pm 20 \mathrm{~mm}$ was analyzed using the slump test. In the hardened state, tensile and compressive strengths were analyzed at 3, 7 and 21 days of hydration. A decrease in the mechanical performance of the concrete was observed when the gravel was replaced by EC. Despite this decrease, lightweight concretes resulted in greater strength/weight ratios. It was observed that the addition of SF significantly improved the mechanical properties of lightweight concrete. Thus, it is possible to conclude that the addition of SF can compensate for the loss of mechanical performance caused by the replacement of conventional coarse aggregate with expanded clay, without the need to increase the amount of cement in the mixture.
\end{abstract}

KEY WORDS: Lightweight concrete; Silica fume; Expanded clay; Efficiency factor. 


\section{INTRODUÇÃO}

Nas últimas décadas, devido à modernização de equipamentos e ao aprofundamento de pesquisas em benefício do concreto, grandes conquistas foram alcançadas como o desenvolvimento de concretos especiais. Eles são utilizados com finalidades específicas, que demandam propriedades que não poderiam ser alcançadas com o concreto convencional (CC). São exemplos desse tipo de concreto, concreto leve $(\mathrm{CL})$, concreto de alto desempenho (CAD), concreto auto-adensável (CAA) e concreto compactado a rolo (CCR) (LIMA et al., 2014; DONATO, 2003; BONIFÁCIO; GODINHO, 2015).

Para a produção do $\mathrm{CL}$, materiais alternativos aos tradicionais como argila expandida, cinzas volantes, xisto, vermiculita, entre outros, são utilizados com o objetivo de diminuir a massa específica do concreto e aumentar a relação resistência/peso, também conhecida como fator de eficiência. A substituição do agregado convencional pela utilização de um material poroso de baixa massa específica, é fundamental para fabricação do CL (MORAVIA, 2010).

Segundo Mo et al. (2017), o CL possui melhores propriedades de isolamento térmico e acústico e possibilita vencer grandes vãos com maior facilidade quando comparado com o CC. Sua densidade é inferior a $2.000 \mathrm{~kg} / \mathrm{m}^{3}$, enquanto a do $\mathrm{CC}$ varia em torno de $2.400 \mathrm{~kg} / \mathrm{m}^{3}$. Essa redução de densidade induz menor carga a estruturas de concreto e consequentemente incide em menores gastos em custos de transporte e manuseio.

A adição de um agregado poroso resulta na diminuição da resistência à compressão do $\mathrm{CL}$ em relação ao CC, sendo necessário aumentar o teor de cimento Portland para compensar a perda de desempenho mecânico. Apesar disso, o uso de cimento está atrelado a um alto custo ambiental devido às altas emissões de dióxido de carbono (CO2) geradas durante sua produção (REVILLACUESTA et al., 2020; KHAN et al., 2020). Substituir parte do cimento em concretos leves por materiais cimentícios suplementares (MCS) se mostra uma alternativa para reduzir consumo de cimento e os problemas ambientais associados à sua produção.

Com o uso de MCS altamente reativos como a sílica ativa (SA) é possível reduzir o impacto ambiental para produção do concreto leve. Esses materiais, ao reagirem com o hidróxido de cálcio $(\mathrm{CH})$ gerado na hidratação do cimento, possibilitam a melhoria nas propriedades do concreto em seus estados fresco e endurecido, através da formação de silicatos de cálcio hidratados (C-S-H) adicionais, reação denominada de pozolânica (FERNANDES, 2018). Com isso, a diminuição do desempenho mecânico do concreto com a utilização de agregados leves pode ser compensada pelo aumento do desempenho mecânico oriundo da reação pozolânica.

A possibilidade de utilizar um material pozolânico é desejável para reduzir o consumo de cimento e a pegada de carbono. Dessa forma, este trabalho teve como objetivo verificar o efeito da substituição da brita por $A E$ e adição de SA na consistência dos concretos leves, assim como, verificar as resistências à tração e à compressão e o fator de eficiência desses concretos.

\section{REVISÃO BIBLIOGRÁFICA 2.1 Concreto leve}

Para atender as necessidades de obras que demandam propriedades especificas, concretos especiais vêm sendo desenvolvidos, como o CAA, o CCR, concreto reforçado com fibras e o CL (LIMA et al., 2014; DONATO, 2003; BONIFÁCIO; GODINHO, 2015). O CL, objeto em estudo no presente trabalho, em geral, têm apresentado massa especifica seca abaixo de $2.000 \mathrm{~kg} / \mathrm{m}^{3}$ e tem como objetivo reduzir o peso das estruturas (SACHT; ROSSIGNOLO; SANTOS, 2010).

A substituição do agregado convencional por agregado de menor densidade incide na redução da massa específica, entretanto, pode ocasionar alterações significativas em outras importantes características do concreto, com destaque significativo na diminuição da resistência mecânica (ROSSIGNOLO; OLIVEIRA, 2005; GOMES et al., 2015).

A NBR 6118 (ABNT, 2014) estabelece a resistência mínima do concreto para utilização estrutural de $20 \mathrm{MPa}$. O American Concrete Institute (Instituto de Concreto Americano), por meio da norma 213R$03(\mathrm{ACl}, 2003)$, indica resistência mínima à compressão aos 28 dias do concreto para utilização estrutural de $17 \mathrm{MPa}$. Segundo Rossignolo et al. (2003), é considerado concreto leve de alto desempenho um concreto com fator de eficiência acima de $25 \mathrm{MPa} . \mathrm{dm}^{3} / \mathrm{kg}$.

$O$ fator de eficiência (FE) pode ser interpretado como um parâmetro da razão entre a resistência à compressão e a massa específica seca do concreto. Esta análise é realizada conforme mostrado na Equação (1) (ROSSIGNOLO et al., 2003; ANGELIN et al., 2014; SCOBAR et al., 2016).

$$
F E=\frac{\text { Resistêneía à comprescằ }}{\text { Massa especifica seca do concreto }}
$$

São muito procurados para diminuir a massa específica seca do concreto materiais como a vermiculita do grupo de minerais de estrutura 
micáceo-lamelar e clivagem basal que expande 20 a 30 vezes a sua espessura inicial, o EPS (poliestireno expandido), mais conhecido como isopor ${ }^{\circledR}$, que possui fácil manuseio e é um excelente isolante e a argila expandida (SUTCU, 2015; STOCCO, 2009).

\subsection{Concreto leve com argila expandida}

A argila expandida é um agregado derivado do aquecimento de uma mistura de diferentes tipos de argilas a temperaturas extremamente elevadas $\left(>1200^{\circ} \mathrm{C}\right)$. Durante o processo de aquecimento, há o aumento de volume do material, cerca de sete vezes o seu tamanho inicial (BONIFÁCIO; GODINHO, 2015).

Durante o processo de calefação da argila, os gases liberados por efeito do aquecimento das misturas de argila acabam sendo incorporados pela massa sinterizada e ficam retidos no interior do agregado. Esse aprisionamento do ar na estrutura celular do agregado contribui de maneira relevante para mudanças significativas nas propriedades térmicas do concreto, contribui para a redução da massa unitária, e concomitantemente, diminui o peso das estruturas de concreto (MORAVIA et al., 2006; SACHT; ROSSIGNOLO; SANTOS, 2010).

A AE é um material bastante procurado para uso no $C L$ devido ao bom desempenho na trabalhabilidade dos concretos. Um exemplo da utilização do material é a execução do tabuleiro da ponte Stovset (1997), construída na Noruega, na qual a sua utilização possibilitou a redução das dimensões dos elementos estruturais e viabilizou o aumento dos vãos (ROSSIGNOLO, 2009; MAYCÁ et al., 2008).

\subsection{Concreto leve com sílica ativa}

A SA ou microssílica é um material em pó fino obtido como subproduto da fabricação do silício metálico e de ligas de ferro silício. Normalmente a SA contém alto teor de $\mathrm{SiO}_{2}$ (>90\%), distribuídos em um diâmetro médio de $0,1 \mu \mathrm{m}$ e área superficial na ordem de 20.000 a $25.000 \mathrm{~m}^{2} / \mathrm{kg}$ (FRAGA et al., 2020; LIMA et al., 2018; VASKE; CAMPAGNOLO; DAL MOLIN, 2010). Esse material possui partículas muito finas, com elevada superfície específica e alta energia superficial.

A SA, quando comparada a outros MCS, é o elemento que possui melhor contribuição para aumentar o desempenho de resistência mecânica nos produtos cimentícios. Este ônus influencia diretamente na criação de uma estrutura compacta, com redução da porosidade e no refinamento da distribuição do tamanho dos poros nos compósitos cimentícios (FRAGA et al., 2020; GUIMARÃES, 2014).
Quando utilizada como MCS, a SA pode agir quimicamente através da reação pozolânica com o hidróxido de cálcio, o que resulta numa quantidade adicional de silicato de cálcio hidratado (C-S-H), proporcionando aumento da resistência mecânica do concreto e acréscimo na resistência ao ataque de agentes agressivos. Também possui ação física com o efeito fíler, devido as partículas da SA possuírem pequena extensão proporcionando maior empacotamento, conforme mostrado na figura 1 (HERMANN et al., 2016; ROMANO et al., 2008; VASKE; CAMPAGNOLO; DAL MOLIN, 2010).

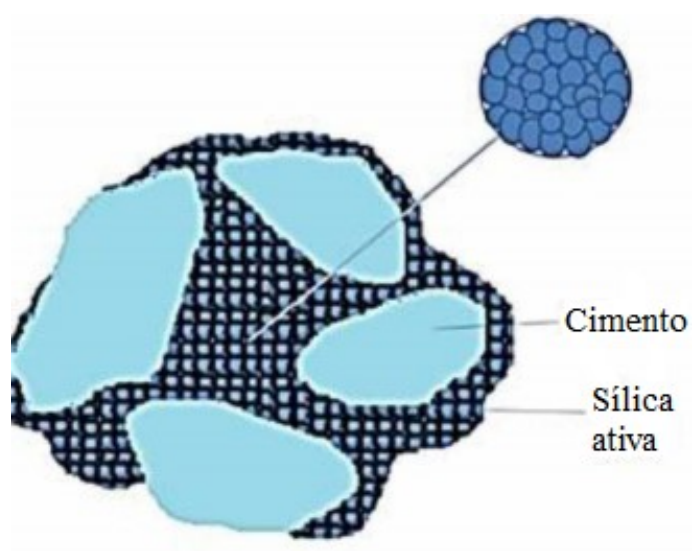

Figura 1. Efeito fíler da SA em materiais cimentícios. Fonte: Sousa, (2017).

Comumente, a utilização da SA varia em teores de 5 até $15 \%$ acerca da massa de cimento. Quando adicionada em teores superiores a $15 \%$, este material deixa de atuar no efeito químico (pozolânico) para atuar apenas com o efeito físico (fíler), o que torna maior a demanda de água devido as partículas muito finas da SA possuírem maior superfície específica que o cimento Portland (HERMANN et al., 2016).

\section{MATERIAIS E MÉTODOS 3.1 Materiais}

Para realização deste trabalho, foram utilizados cimento Portland de alta resistência inicial (CPVARI) Cauê - Grupo Intercement, aditivo superplastificante Tecflow $50 \mathrm{~N}$ da Grace e sílica ativa da Elkem. Os agregados utilizados foram areia natural quartzosa, brita zero, e argila expandida tipo 2215 da Cinexpan. A água utilizada foi da Companhia de Saneamento Ambiental do Distrito Federal (CAESB). No quadro 1 são mostradas as propriedades dos materiais utilizados e na figura 2 são apresentadas as curvas de distribuição granulométrica dos agregados miúdos e graúdos.

0 agregado miúdo utilizado corresponde aos limites de zona utilizável em concretos, atendendo aos pré-requisitos da ABNT NBR 7211 (2009), conforme pode-se observar na figura 2(a). 

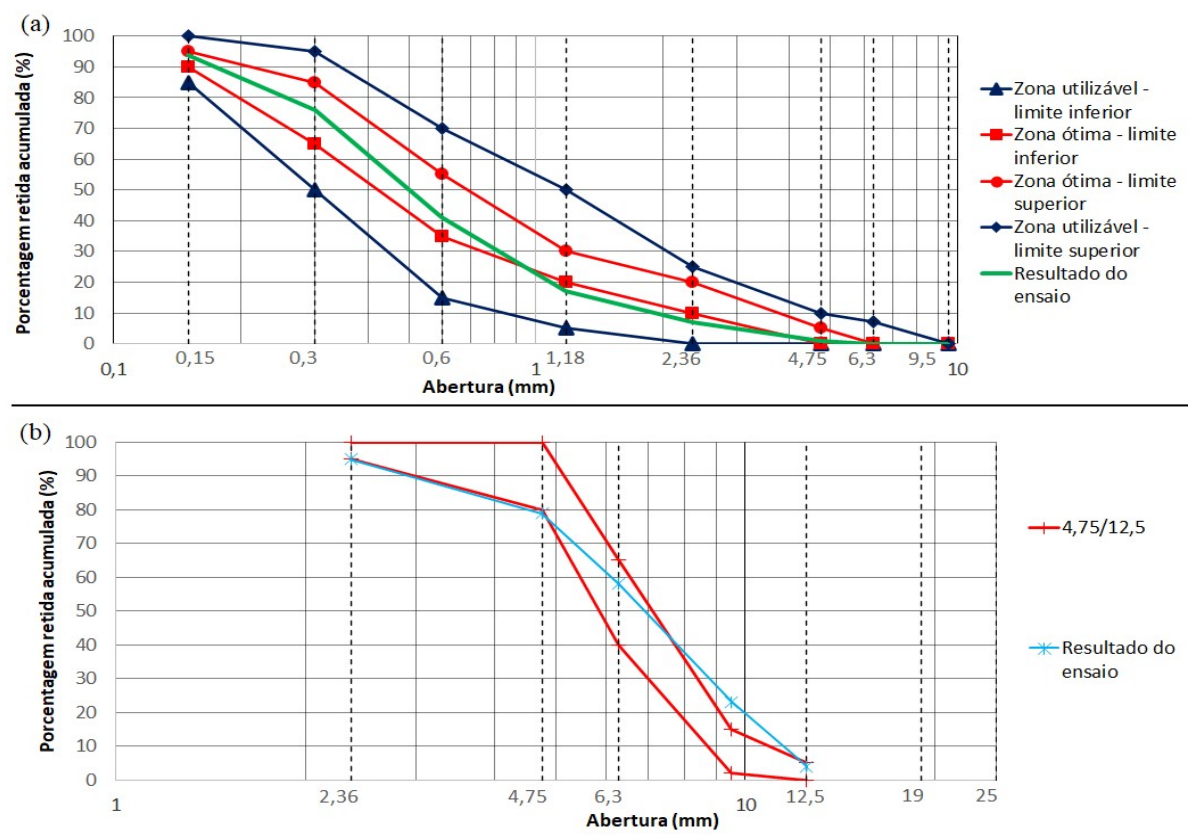

Figura 2. Curva granulométrica do agregado (a) miúdo; (b) graúdo. Fonte: autores.

QUADRO 1: Propriedades dos materiais utilizados

\begin{tabular}{|c|c|c|c|}
\hline MATERIAL & \multicolumn{2}{|c|}{ PROPRIEDADES } & VALOR \\
\hline \multirow{4}{*}{ CP V - ARI } & \multicolumn{2}{|c|}{ Massa específica $\left(\mathrm{g} / \mathrm{cm}^{3}\right)$} & 3,15 \\
\hline & \multicolumn{2}{|c|}{ Finura por meio da peneira $75 \mu \mathrm{m}(\%)$} & 2,00 \\
\hline & \multirow{2}{*}{ Tempos de pega } & Início (min) & 115,0 \\
\hline & & Fim (min) & 204,0 \\
\hline \multirow{3}{*}{$\begin{array}{l}\text { Agregado } \\
\text { miúdo }\end{array}$} & \multicolumn{2}{|c|}{ Dimensão máxima característica (mm) } & 4,75 \\
\hline & \multicolumn{2}{|c|}{ Módulo de finura (\%) } & 3,34 \\
\hline & \multicolumn{2}{|c|}{ Massa específica $\left(\mathrm{g} / \mathrm{cm}^{3}\right)$} & 2,65 \\
\hline \multirow{3}{*}{$\begin{array}{l}\text { Agregado } \\
\text { graúdo (brita } \\
0 \text { ) }\end{array}$} & \multicolumn{2}{|c|}{ Dimensão máxima característica (mm) } & 12,5 \\
\hline & \multicolumn{2}{|c|}{ Módulo de finura (\%) } & 5,85 \\
\hline & \multicolumn{2}{|c|}{ Massa específica $\left(\mathrm{g} / \mathrm{cm}^{3}\right)$} & 1,41 \\
\hline \multirow{4}{*}{$\begin{array}{l}\text { Agregado } \\
\text { graúdo (argila } \\
\text { expandida) }\end{array}$} & \multicolumn{2}{|c|}{ Classes granulométricas $(\mathrm{cm})$} & $\begin{array}{l}1,50- \\
2,20\end{array}$ \\
\hline & \multicolumn{2}{|c|}{ Densidade aparente $\left(\mathrm{kg} / \mathrm{m}^{3}\right)$} & $500 \pm 10$ \\
\hline & \multicolumn{2}{|c|}{ Massa específica $\left(\mathrm{kg} / \mathrm{dm}^{3}\right)$} & 0,64 \\
\hline & \multicolumn{2}{|c|}{ Massa unitária $\left(\mathrm{g} / \mathrm{cm}^{3}\right)$} & 0,49 \\
\hline \multirow{3}{*}{$\begin{array}{l}\text { Aditivo } \\
\text { superplastific } \\
\text { ante }\end{array}$} & \multicolumn{2}{|c|}{ Massa específica $\left(\mathrm{g} / \mathrm{cm}^{3}\right)$} & $\begin{array}{l}1,090- \\
1,130\end{array}$ \\
\hline & \multicolumn{2}{|c|}{ Aspecto/cor } & Alaranjado \\
\hline & \multicolumn{2}{|c|}{ Dosagem recomendada (\% SPC) } & $\begin{array}{c}0,30- \\
1,20 \\
\end{array}$ \\
\hline \multirow{2}{*}{ Sílica ativa } & \multicolumn{2}{|c|}{ Massa específica $\left(\mathrm{g} / \mathrm{cm}^{3}\right)$} & 2,20 \\
\hline & \multicolumn{2}{|c|}{ Densidade aparente em estado solto $\left(\mathrm{kg} / \mathrm{m}^{3}\right)$} & 682,7 \\
\hline
\end{tabular}

O mesmo encontra-se quase em sua totalidade dentro da zona ótima. Observa-se na figura 2(b), que o agregado graúdo se encontra na faixa de $4,75 \mathrm{~mm} / 12,5 \mathrm{~mm}$ (menor/maior dimensão), com ensaio executado de acordo com a metodologia da NBR 7211 (ABNT, 2009).

\subsection{Preparação dos concretos}

A definição dos traços de concreto foi realizada buscando-se analisar o efeito da substituição volumétrica do agregado graúdo natural por agregado graúdo leve de argila expandida. A nomenclatura dos traços adotados é apresentada no quadro 2.

QUADRO 2: Nomenclatura e composição dos traços

\begin{tabular}{|c|l|}
\hline CONCRETO & \multicolumn{1}{|c|}{ COMPOSIÇÃO } \\
\hline REF & Concreto convencional. \\
\hline $50 \mathrm{~B} 50 \mathrm{AE}$ & $\begin{array}{l}\text { Concreto com substituição de 50\% da brita por } \\
\text { argila expandida. }\end{array}$ \\
\hline 50B50AE10SA & $\begin{array}{l}\text { Concreto com substituição de 50\% da brita por } \\
\text { argila expandida e adição de 10\% de sílica } \\
\text { ativa. }\end{array}$ \\
\hline 100AE & $\begin{array}{l}\text { Concreto com substituição de 100\% da brita } \\
\text { por argila expandida. }\end{array}$ \\
\hline 100AE10SA & $\begin{array}{l}\text { Concreto com substituição de 100\% da brita } \\
\text { por argila expandida e adição de } 10 \% \text { de sílica } \\
\text { ativa. }\end{array}$ \\
\hline
\end{tabular}

Para a definição do proporcionamento dos materiais utilizados em cada traço, foi utilizada a metodologia adaptada de Helene e Terzian (1992). Por meio dessa metodologia, chegou-se ao traço do concreto REF igual a $1: 1,57: 1,93$, com relação água/cimento igual a 0,45 e teor de aditivo 
superplastificante igual a $0,40 \%$ em relação à massa de cimento. Dessa forma, todos os concretos seguiram esse mesmo traço, havendo apenas a substituição, em volume, da brita pela argila expandida e/ou a adição, em massa, de 10\% de sílica ativa. A relação a/c foi mantida constante para não influenciar no desempenho mecânico dos concretos. Além disso, foi fixada a consistência dos concretos em $100 \pm 20 \mathrm{~mm}$ no ensaio de abatimento do tronco de cone, realizado conforme a NBR 67 (ABNT, 1998). Para isso, foi variado o teor de aditivo superplastificante de cada concreto estudado. O quantitativo dos materiais utilizados para moldagem de seis corpos de prova cilíndricos com $100 \mathrm{~mm}$ de diâmetro e $200 \mathrm{~mm}$ de altura pode ser observado no quadro 3.

QUADRO 3: Quantitativo para moldagem de seis corpos de prova cilíndricos de concreto com $100 \mathrm{~mm}$ de diâmetro e $200 \mathrm{~mm}$ de altura.

\begin{tabular}{|c|c|c|c|c|c|c|c|c|c|}
\hline Concreto & \multirow{2}{*}{$\begin{array}{c}\text { Cimento } \\
(\mathbf{k g})\end{array}$} & $\begin{array}{c}\text { Areia } \\
(\mathbf{k g})\end{array}$ & $\begin{array}{c}\text { Brita } \\
(\mathbf{k g})\end{array}$ & $\begin{array}{c}\text { Argila } \\
\text { expandida } \\
(\mathbf{k g})\end{array}$ & $\begin{array}{c}\text { Sílica } \\
\text { ativa } \\
(\mathbf{k g})\end{array}$ & $\begin{array}{c}\text { Água } \\
(\mathbf{k g})\end{array}$ & $\begin{array}{c}\text { Aditivo } \\
\text { Superplastificante }\end{array}$ & $\begin{array}{c}\text { Abatimento } \\
\text { do tronco de } \\
\text { cone (mm) }\end{array}$ \\
\hline REF & 7,0 & 10,99 & 13,51 & - & - & 3,15 & 0,40 & 28,0 & 80,0 \\
\hline 50B50AE & 7,0 & 10,99 & 6,80 & 2,34 & - & 3,15 & 0,20 & 14,0 & 80,0 \\
\hline 50B50AE10SA & 7,0 & 10,99 & 6,80 & 2,34 & 0,70 & 3,15 & 0,55 & 38,50 & 90,0 \\
\hline 100AE & 7,0 & 10,99 & - & 4,68 & - & 3,15 & 0,25 & 17,50 & 95,0 \\
\hline 100AE10SA & 7,0 & 10,99 & - & 4,68 & 0,7 & 3,15 & 0,55 & 38,50 & 80,0 \\
\hline
\end{tabular}

Com o teor de aditivo superplastificante definido, foram moldados 90 corpos de prova cilíndricos. Após a moldagem e decorridas 24 horas cobertos com uma lona plástica para evitar a perda de água do concreto por evaporação, os corpos de prova foram desmoldados e colocados submersos em água saturada de cal até a idade da determinação das resistências à tração e à compressão, aos 3, 7 e 21 dias de hidratação.

\subsection{Resistências à tração e à compressão dos concretos}

A verificação da resistência à tração por compressão diametral foi realizada conforme determinações da ABNT NBR 7222 (2011) e a verificação da resistência à compressão foi regulamentada pela ABNT NBR 5739 (2018).

Em ambos ensaios, três corpos de prova de cada traço foram capeados e rompidos em uma máquina universal para ensaios mecânicos nas idades de 3, 7 e 21 dias de hidratação. A tolerância de tempo para rompimento dos concretos foi respeitada de acordo com as recomendações da ABNT NBR 5739 (2018).

A partir dos resultados obtidos na realização dos dois ensaios, precedeu-se uma análise estatística com o software STATÍSTICA v10, em que cada traço foi testado a um nível de significância $(\boldsymbol{\alpha})$ de 0,05 por idade avaliada. Foi realizada uma análise de variância ANOVA dos dados dos ensaios de tração e compressão, com o intuito de identificar se as resistências dos traços diferem de maneira significativa. Essa influência é indicada por meio da probabilidade significativa ( $\mathrm{p}$-valor), na qual para os resultados significativos o $p$-valor não deve ser maior que o nível de significância $(\boldsymbol{\alpha})$. Caso o pvalor seja igual ou superior a 0,05 , o resultado é considerado não significativo, ou seja, as resistências são estatisticamente iguais.

Em seguida, com o auxílio do software STATÍSTICA v10, foi realizado o teste de Duncan, na qual os dados gerados foram submetidos à uma comparação múltipla entre si, separando-os por classes homogêneas de resistências, levando em consideração a média e o desvio padrão das duas resistências para cada ensaio separadamente, a um nível de confiança de $95 \%$.

\subsection{Fator de eficiência}

Foi realizado o cálculo do fator de eficiência, conforme mostrado na Equação (1), para relacionar a resistência mecânica com a massa específica dos concretos. Os valores de resistência mecânica utilizados foram os resultados das médias de cada idade avaliada e a massa específica considerada foi resultado do valor médio das massas dos concretos estudados aos 21 dias de hidratação.

\section{RESULTADOS}

\subsection{Propriedades no estado fresco}

Foi realizado o ensaio de abatimento do tronco de cone para verificar se o abatimento atingiu o valor predeterminado de $100 \pm 20 \mathrm{~mm}$. Na figura 3 é mostrado o procedimento de verificação do abatimento dos concretos, sequencialmente, do primeiro (REF) ao último traço (100AE10SA). 


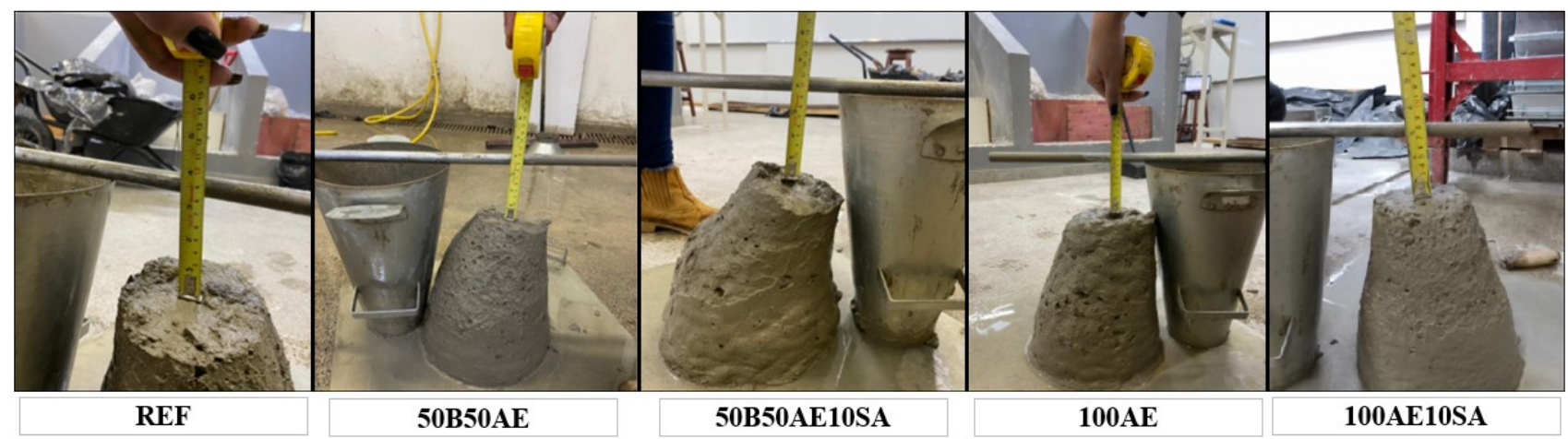

Figura 3. Ensaio de abatimento do tronco de cone.

Como pode ser observado na Figura 3 e no Quadro 3, a adição de $A E$ melhorou a fluidez do concreto, diminuindo a necessidade de aditivo superplastificante, resultado do formato arredondado do agregado, que proporciona efeito de rolagem e melhora a trabalhabilidade do concreto (ROSSIGNOLO, 2009). Por outro lado, a adição de SA tornou o concreto menos fluido, sendo necessário adicionar mais aditivo superplastificante, por causa da elevada superfície específica do material, que torna maior o consumo de água (HERMANN et al., 2016).

Os resultados de resistência média à tração dos concretos aos 3, 7 e 21 dias de hidratação são mostrados na figura 4.

Nas idades de 3 e 7 dias, no que se refere à resistência à tração, notou-se que todos os concretos apresentaram similaridade nos resultados. Aos 21 dias de hidratação, o traço 50B50AE apresentou média similar ao REF e o traço 50B50AE10SA apresentou aumento de 8,70\% em relação ao REF, mostrando que a SA contribuiu para

o aumento da resistência à tração do concreto. Os traços 100AE e 100AE10SA apresentaram uma redução de 34,80\% em relação ao REF. Segundo Rossignolo et al. (2003) o grande volume de vazios proporcionados pelos agregados leves em relação aos agregados convencionais, com até $50 \%$ do volume total para argilas expandidas, é o fator responsável pela diminuição da resistência à tração dos concretos, o que corrobora com os resultados em que houveram redução de resistência mecânica.

Considerando-se a média dos resultados à tração das três idades analisadas, a adição de SA nos traços 50B50AE10SA e 100AE10SA aumentou em média $10 \%$ a resistência à tração dos concretos em relação aos traços 50B50AE e 100AE. Esse aumento de resistência justifica-se pela reação pozolânica, que resulta numa quantidade adicional de $\mathrm{C}-\mathrm{S}-\mathrm{H}$ (HERMANN et al., 2016; ROMANO et al., 2008; VASKE; CAMPAGNOLO; DAL MOLIN, 2010).

De acordo com a análise de variância (one-way ANOVA) da resistência à tração, o p-valor foi equivalente a 0,304180 para 3 dias, e 0,543385 para 7 dias de hidratação, confirmando que os dados não diferem entre si de maneira significativa.

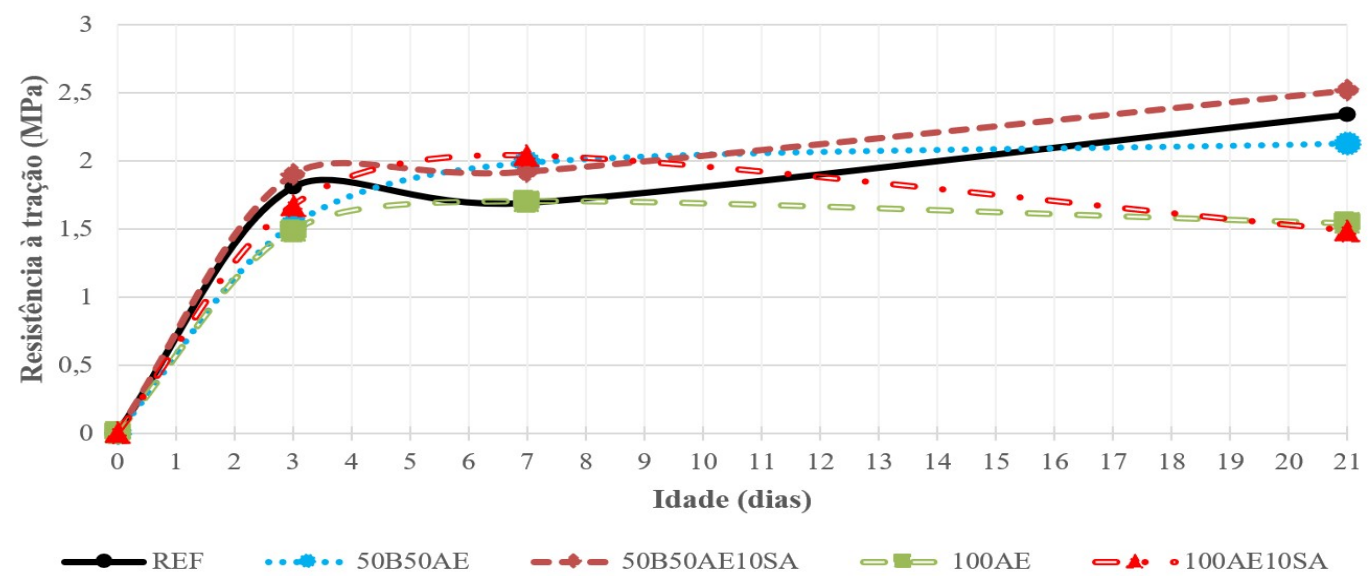

Figura 4. Resistência média à tração dos concretos aos 3, 7 e 21 dias de hidratação 
Aos 21 dias, o p-valor foi equivalente a 0,020088. Dessa forma, como o p-valor foi menor do que o nível de significância $(0,05)$, houve diferença significativa na resistência à tração entre os concretos aos 21 dias de hidratação. Com isso, para comparar os dados de resistência à tração dos concretos aos 21 dias, foi realizada uma análise estatística através do teste de Duncan, como mostrado no quadro 4.

Foram formados 2 grupos de resistência à tração dos concretos, nos quais foi possível observar que os concretos REF, 50B50AE e 50B50AE10SA resultaram em maior resistência à tração que os demais, pois foram classificados no grupo 2 (de maior resistência). Os traços com $100 \%$ de argila expandida (100AE e 100AE10SA), resultaram em média $53 \%$ de redução da resistência à tração, em relação à média dos traços classificados no grupo 2 , devido os traços com argila expandida possuírem maior índice de vazios (até $50 \%$ do volume total), conforme (ROSSIGNOLO e OLIVEIRA, 2007).

\subsection{Resistência à compressão dos concretos}

Os resultados de resistência média à compressão dos concretos aos 3, 7 e 21 dias de hidratação são mostrados na figura 5 .

Aos 7 dias, os concretos REF e 50B50AE10SA apresentaram maiores resistências à compressão. Entre 7 e 21 dias de hidratação, o concreto 50B50AE10SA apresentou maior aumento de resistência (27\%) em relação aos demais, resultando na maior resistência média à compressão aos 21 dias de hidratação com 41,5 $\mathrm{MPa}$.

QUADRO 4. Classificação por grupos de resistência à tração dos concretos aos 21 dias de hidratação

\begin{tabular}{|c|c|c|c|c|c|}
\hline \multirow{3}{*}{ Idade } & Concreto & $\begin{array}{c}\text { Resistência média à } \\
\text { tração (MPa) }\end{array}$ & Desvio padrão (MPa) & Grupo 1 & Grupo 2 \\
\hline \multirow{5}{*}{21 dias } & REF & 2,34 & 0,30 & & $\mathrm{x}$ \\
\cline { 2 - 6 } & 50B50AE & 2,13 & 0,60 & $\mathrm{x}$ & $\mathrm{x}$ \\
\cline { 2 - 6 } & 50B50AE10SA & 2,52 & 0,40 & $\mathrm{x}$ & \\
\cline { 2 - 6 } & $100 \mathrm{AE}$ & 1,54 & 0,20 & & $\mathrm{x}$ \\
\hline
\end{tabular}

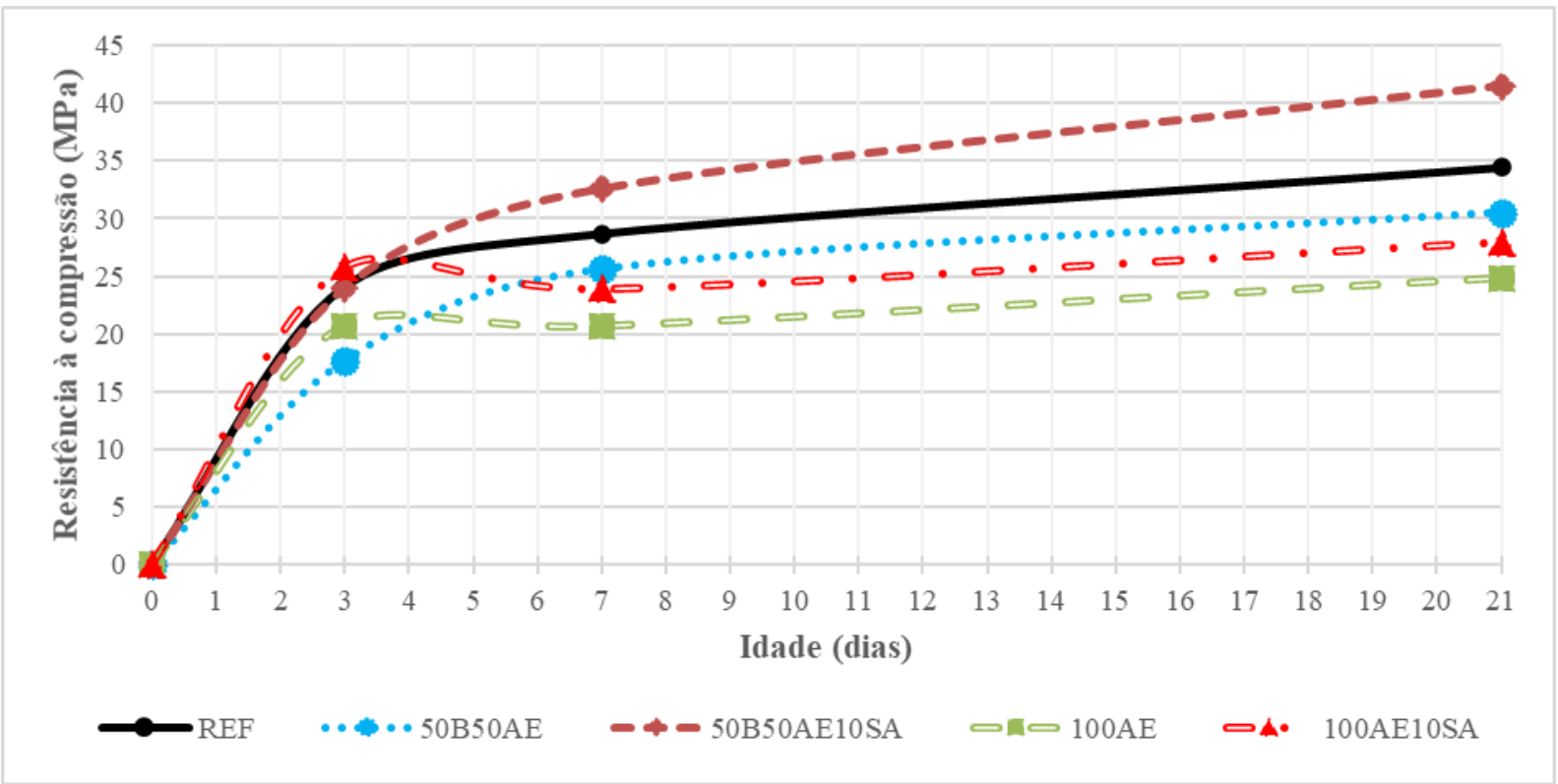

Figura 5. Resistência média à compressão dos concretos aos 3, 7 e 21 dias de hidratação 
Foi possível observar, na análise de variância (oneway ANOVA), que aos 3 dias de hidratação o p-valor foi igual a 0,001117 , aos 7 dias o $p$-valor foi igual a 0,00822 e aos 21 dias o resultado do $p$-valor foi igual a 0,000309, ou seja, todos foram menores que o nível de significância de 0,05 e, consequentemente, a resistência à compressão dos concretos diferiram de maneira significativa nas três idades avaliadas.

Dessa forma, com o auxílio do software STATÍSTICA $\mathrm{v} 10$, foi realizado o teste de Duncan, no qual os dados de resistência à compressão foram submetidos à uma comparação múltipla entre si, conforme mostrado no quadro 5 .

Aos 3 dias de hidratação foram formados 2 grupos de resistência, sendo que o grupo 1 corresponde à menor resistência e o grupo 2 à maior resistência. A resistência à compressão dos traços 100AE10SA e 50B50AE10SA apresentaram valores similares à resistência à compressão do traço REF, enquanto os traços 50B50AE e 100AE apresentaram redução de resistência à compressão, em comparação com o traço REF. Como o agregado leve é mais poroso que o agregado convencional, a ruptura ocorre através da própria argila expandida, que não suporta os esforços mecânicos, conforme mostrado na figura 6.

QUADRO 5 - Classificação por grupos de resistência à compressão dos concretos aos 3, 7 e 21 dias de hidratação

\begin{tabular}{|c|c|c|c|c|c|c|c|}
\hline Idade & Concreto & $\begin{array}{l}\text { Resistência média à } \\
\text { compressão (MPa) }\end{array}$ & $\begin{array}{l}\text { Desvio } \\
\text { padrão } \\
\text { (MPa) }\end{array}$ & Grupo 1 & Grupo 2 & Grupo 3 & Grupo 4 \\
\hline \multirow{5}{*}{3 dias } & REF & 24,1 & 1,1 & & $\mathrm{x}$ & & \\
\hline & 50B50AE & 17,7 & 2,5 & $\mathrm{x}$ & & & \\
\hline & 50B50AE10SA & 23,9 & 1,9 & & $\mathrm{x}$ & & \\
\hline & $100 \mathrm{AE}$ & 20,7 & 0,1 & $\mathrm{x}$ & & & \\
\hline & 100AE10SA & 25,7 & 1,7 & & $\mathrm{x}$ & & \\
\hline \multirow{5}{*}{7 dias } & REF & 28,7 & 1,4 & & & $\mathrm{x}$ & $\mathrm{x}$ \\
\hline & 50B50AE & 25,6 & 2,5 & & $\mathrm{x}$ & $\mathrm{x}$ & \\
\hline & 50B50AE10SA & 32,6 & 1,9 & & & & $\mathrm{x}$ \\
\hline & $100 \mathrm{AE}$ & 20,6 & 3,6 & $\mathrm{x}$ & & & \\
\hline & 100AE10SA & 23,9 & 1,3 & $\mathrm{x}$ & $\mathrm{x}$ & & \\
\hline \multirow{5}{*}{21 dias } & REF & 34,4 & 4,7 & & & $\mathrm{x}$ & \\
\hline & $50 \mathrm{~B} 50 \mathrm{AE}$ & 30,5 & 2,9 & & $\mathrm{x}$ & $\mathrm{x}$ & \\
\hline & 50B50AE10SA & 41,5 & 1,1 & & & & $\mathrm{x}$ \\
\hline & $100 \mathrm{AE}$ & 24,8 & 2,1 & $\mathrm{x}$ & & & \\
\hline & 100AE10SA & 27.9 & 1,1 & $\mathrm{x}$ & $\mathrm{x}$ & & \\
\hline
\end{tabular}

Observação: Os grupos são independentes entre as idades. Dessa forma, os grupos de uma idade não estão relacionados com os grupos das demais idades.

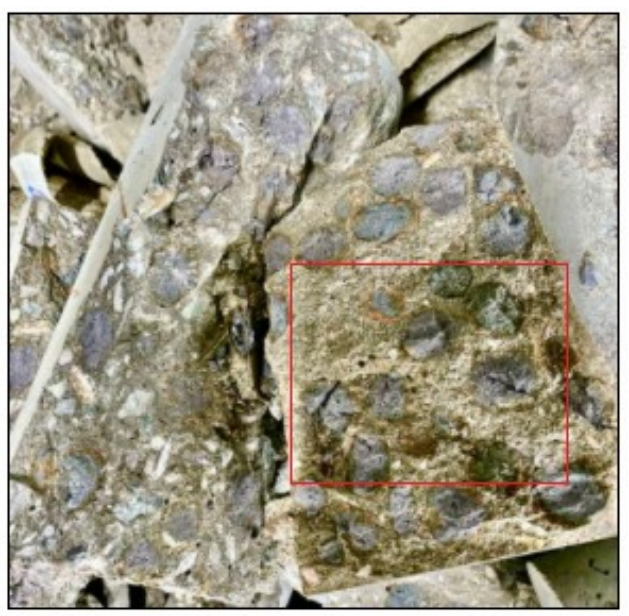

Figura 6. Rompimento do concreto na argila expandida.
Aos 7 dias foram formados 4 grupos de resistência. Foi observado que o traço $100 \mathrm{AE}$ resultou na menor resistência à compressão entre os concretos avaliados. O traço 100AE10SA obteve uma redução de $8 \%$ de 3 para 7 dias de hidratação. Essa redução é resultado da maior presença de argila nos corpos de prova, o que tornou possível o aumento do índice de vazios e concomitantemente, a redução da resistência à compressão. Conforme Mehta e Monteiro (2008), a combinação da espessura e qualidade da zona de transição influencia diretamente na resistência mecânica, o que corrobora com os resultados verificados para 0 traço 100AE10SA. O traço 50B50AE obteve aumento de resistência em torno de $44 \%$, enquanto o traço 50B50AE10SA em torno de $36 \%$, superando o traço REF. Borja (2011), associa esse aumento da 
resistência mecânica à diminuição da zona de transição entre agregado e pasta de cimento provocada pela ligação da argila expandida com a sílica ativa.

Aos 21 dias também foram formados 4 grupos de resistência. Todos os concretos apresentaram aumento de resistência à compressão em relação às idades anteriores. Esse aumento de resistência à compressão representa uma porcentagem de $43 \%$ em relação aos dados obtidos aos 3 dias de hidratação e $20 \%$ em relação aos dados obtidos aos 7 dias de hidratação. Segundo a NBR 6118 (ABNT, 2014), a resistência mínima do concreto para utilização estrutural é de $20 \mathrm{MPa}$. Já a ACI 213R-03 (2003), a resistência mínima à compressão aos 28 dias do concreto para utilização estrutural é de 17 $M P a$. Nesse trabalho, a menor média de resistência à compressão aos 21 dias é apresentada pelo traço $100 \mathrm{AE}$, com valor de 24,8 MPa, consolidando a utilização de todos os concretos estudados para uso estrutural.

\subsection{Fator de eficiência}

Os resultados do fator de eficiência dos concretos são mostrados no quadro 6 .

QUADRO 6. Fator de eficiência dos concretos.

\begin{tabular}{|c|c|c|c|c|}
\hline \multirow{2}{*}{ Concreto } & \multirow{2}{*}{ Densidade $\left(\mathbf{k g} / \mathbf{d m}^{\mathbf{3}}\right.$ ) } & \multicolumn{3}{|c|}{ Fator de Eficiência (MPa.dm $\mathbf{M g}^{\mathbf{3}}$} \\
\cline { 3 - 5 } & & $\mathbf{3}$ & $\mathbf{2}$ \\
\cline { 3 - 5 } & & 10,25 & 12,20 & 14,64 \\
\hline REF & 2,35 & 8,96 & 13,00 & 15,49 \\
\hline 50B50AE & 1,97 & 12,01 & 16,34 & 20,80 \\
\hline 50B50AE10SA & 1,99 & 12,76 & 12,73 & 15,29 \\
\hline 100AE & 1,62 & 15,46 & 14,35 & 16,77 \\
\hline
\end{tabular}

Quanto aos valores obtidos no ensaio de massa específica seca, a média dos resultados do concreto REF foi de $2.349 \mathrm{~kg} / \mathrm{m}^{3}$. O traço 50B50AE obteve média de $1.971 \mathrm{~kg} / \mathrm{m}^{3}$ e o traço 50B50AE10SA, obteve média de $1.994 \mathrm{~kg} / \mathrm{m}^{3}$, reduzindo $19 \%$ e $17,8 \%$, respectivamente, o peso do concreto REF, enquanto os concretos 100AE e 100AE10SA, com $100 \%$ de argila expandida, obtiveram média de $1.621 \mathrm{~kg} / \mathrm{m}^{3}$ e $1.666 \mathrm{~kg} / \mathrm{m}^{3}$, com redução média de $40 \%$ da massa específica seca em relação ao concreto REF. A massa específica aparente dos concretos com argila expandida obteve resultados inferiores a $2.000 \mathrm{~kg} / \mathrm{m}^{3}$, o que torna possível a classificação de todos os concretos com substituição parcial e total do agregado graúdo por agregado leve, como concretos leves.

Aos 3 dias, é possível observar que o fator de eficiência do traço 50B50AE é inferior ao REF. Essa redução de $14 \%$ é resultado da maior presença de argila nos corpos de prova, o que tornou possível o aumento do índice de vazios e concomitantemente, a redução da resistência à compressão para a idade avaliada. Nas demais idades, é possível observar que todos os traços apresentaram maiores fatores de eficiência do que o concreto REF. Essa característica é facilmente visualizada quando levada em consideração a menor massa específica pertinente dos concretos leves.

\section{CONCLUSÃO}

A partir dos resultados experimentais, ficou evidente que a substituição total do agregado graúdo convencional por um agregado leve, de menor densidade, não confere efeitos positivos ao concreto quando comparado ao traço convencional. Entretanto, a partir do acréscimo da sílica ativa no teor de $10 \%$, apesar da redução dos valores de resistência, há um aumento significativo do fator de eficiência em relação ao concreto referência.

Os concretos pesquisados contendo argila expandida, com ou sem sílica ativa, podem ser considerados leves devido à massa específica seca ter apresentado resultados inferiores a 2.000 $\mathrm{kg} / \mathrm{m}^{3}$. A adição de sílica ativa aumentou 0 desempenho mecânico dos concretos leves sem ser necessário incorporar um maior teor de cimento Portland, resultando em menores impactos ambientais para produção do concreto leve.

Dessa forma concluiu-se que a argila expandida como agregado leve é uma alternativa viável para preparação de concretos, sendo recomendada a sua 
utilização com sílica ativa para melhoria das propriedades mecânicas.

\section{REFERÊNCIAS}

ASSOCIAÇÃO BRASILEIRA DE NORMAS TÉCNICAS, ABNT. NBR 7211 - Agregados para concreto Especificação. Rio de Janeiro, 2009.

ANGELIN, A. F. Concreto leve estrutural Desempenhos físicos, térmicos, mecânicos e microestruturais. 2014. p. 1-98. Dissertação (Mestrado em Tecnologia) - Tecnologia e Inovação. Universidade Estadual de Campinas, Limeira, 2014.

BONIFÁCIO, J. S. R.; GODINHO, D. S. S. Estudo do efeito das fibras de vidro e polipropileno nas propriedades mecânicas do concreto. 2015. $19 \mathrm{f}$. Trabalho de conclusão de curso (Bacharelado em Engenharia Civil) - Universidade do Extremo Sul Catarinense, Criciúma, 2015.

BORJA, E.V. Efeito da adição da argila expandida e adições minerais na formulação de concretos estruturais leves autoadensáveis. 2011. p. 1-230. Tese (Doutorado em Engenharia Mecânica) Engenharia Mecânica. Universidade Federal do Rio Grande do Norte, Natal, 2011.

DONATO, M. Comportamento mecânico de concreto compactado com rolo reforçado com fibras de polipropileno. 2003. p. 1-82. Dissertação (Mestrado em Engenharia) - Engenharia Civil. Universidade Federal do Rio Grande do Sul, Porto Alegre, 2003.

FÉDÉRATION INTERNATIONALE DU BÉTON (FIB). Lightweigh aggregate concrete. Guidance Documents - Bulletin 8. FIB, Lousanne, Suiça, 2000.

FERNANDES, A. J. M. Influência do teor de sílica e alumina no comportamento pozolânico de materiais cimentícios suplementares. 2018. $165 \mathrm{f}$. Dissertação (Mestrado em Engenharia) - Engenharia Civil. Universidade do Vale do Rio dos Sinos, Programa de Pós-Graduação, São Leopoldo, RS, 2018.

FRAGA, Y. S. B.; RÊGO, J. H. S.; CAPUZZO, V. M. S.; ANDRADE, D. S. Efeito da ultrasonicação da sílica ativa e da nanossílica coloidal em pastas de cimento. Revista Matéria, v. 25, n.4, p. 1-16, 2020.

GOMES, P. C. C.; ALENCAR, T. F. F. De; SILVA, N. V. Da; MORAES, K. A. de M.; ANGULO, S. C. Obtenção de concreto leve utilizando agregados reciclados. Ambiente Construído, v. 15, n. 3, p. 31-46, 2015.

GUIMARÃES, J. P. F. Sinergia entre a nanosílica e a sílica ativa na produção do concreto. In: $56^{\circ}$ Congresso Brasileiro do Concreto, 2014, Natal. Anais [...]. Natal: Ibracon, 2014.
HELENE, P., TERZIAN, P. Manual de dosagem e controle do concreto, São Paulo, PINI. SENAI., 1992.

HERMANN, A.; LANGARO, E. A.; SILVA, S. H. L.; KLEIN, N. S. Empacotamento de partículas de cimento e ślica ativa em pastas pelo uso de modelo analítico. Revista IBRACON de Estruturas e Materiais, v. 9, n. 1, p. 48-65, 2016.

LIMA, C. I. V.; COUTINHO, C. O. D.; AZEVEDO, G. G. C.; BARROS, T. Y. G.; TAUBER, T. C.; LIMA, S. F. Concreto e suas inovações. Ciências exatas e tecnológicas, p. 31-40, 2014.

KALPANA, M.; TAYU, A. Experimental investigation on lightweight concrete added with industrial waste (steel waste). Materials Today: Proceedings, v. 22. p. 887-889, 2019.

KHAN, M. N. N.; SAHA, A. K.; SARKER, P. K. Reuse of waste glass as a supplementary binder and aggregate for sustainable cement-based construction materials: A review. Journal of Building Engineering, v. 28, p. 101052, 2020.

LIMA, B. L. N.; OLIVEIRA, M. S.; OLIVEIRA, P. T.; SILVA, L. Análise do potencial da sílica ativa na resistência à compressão compressão do concreto. In: 59 a edição Congresso Brasileiro do Concreto. 2017, p. 0-11, 2018.

MAYCÁ, Jefferson.; CREMONINI, Ruy A.; RECENA, Fernando A. P. Conctribuição ao estudo da argila expandida nacional como alternativa de agregado graúdo para concretos leves estruturais (CLE). 2018.

MEHTA, P.K.; MONTEIRO, P.J.M. Concreto: Microestrutura, Propriedades e Materiais. $1^{\text {a }}$ Edição, São Paulo, IBRACON, 2008.

MO, K. H.; LEE, H. J.; LIU, M. Y. J.; LING, T. C. Incorporation of expanded vermiculite lightweight aggregate in cement mortar. Construction and Building Materials, v. 179, p. 302-306, 2018.

MO, K. H.; LING, T. C.; ALENGARAM, U. J.; YAP, S. P.; YUEN, C. W. Overview of supplementary cementitious materials usage in lightweight aggregate concrete. Construction and Building Materials, v. 139, p. 403-418, 2017.

MORAVIA, W. G; GUMIERI, A. G.; VASCONCELOS, W. L. Efficiency factor and modulus of elasticity of lightweight concrete with expanded clay aggregate. Revista IBRACON de Estruturas e Materiais. São Paulo, v. 3, n. 2, p. 195-204, junho de 2010.

MORAVIA, W. G.; OLIVEIRA, C. A. S.; GUMIERI, A. G.; VASCONCELOS, W. L. Caracterização microestrutural da argila expandida para aplica,ão como agregado em concreto estrutural leve. Ceramica, v. 52, n. 322, p.193-199, 2006. 
REVILLA-CUESTA, V.; SKAF, M.; FALESCHINI, F.; MANSO, J. M.; ORTEGA-LÓPEZ, V. Self-compacting concrete manufactured with recycled concrete aggregate: An overview. Journal of Cleaner Production, v. 262, p. 1-19, 2020.

ROMANO, R. C. O.; SCHREURS, H.; JOHN, V. M.; PILEGGI, R. G. Influência da técnica de dispersão nas propriedades de sílica ativa. Ceramica, v. 54, n. 332, p. 456-461, 2008.

ROSSIGNOLO, J. A.; OLIVEIRA, I. L. Concreto leve estrutural com metacaulim. Revista Minerva. v. 3, n. 2, p. 177-187, 2005.

ROSSIGNOLO, J. A.; OLIVEIRA, I. L. Efeito do Metacaulim nas propriedades do concreto leve estrutural. Acta Scientiarum - Technology, v.29, 2007.

ROSSIGNOLO, J. A. Concreto leve estrutural: produção, propriedades, microestrutura e aplicações. 1 ed. São Paulo: PINI, 2009.

ROSSIGNOLO, J. A. Concreto leve de alto desempenho modificado com SB para préfabricados esbeltos - Dosagem, produção, propriedades e mocroestrutura. 2003. p. 1-211. Tese (Doutorado em Interunidades em Ciência e Engenharia de Materiais) - Engenharia Civil, Universidade de São Paulo, São Paulo, 2003.

ROSSIGNOLO, J. A.; Oliveira, I. L. Efeito do Metacaulim nas propriedades do concreto leve estrutural. Acta Scientiarum - Technology, v.29, 2007.

SACHT, H. M.; ROSSIGNOLO, J. A.; SANTOS, W. N. Avaliação da condutividade térmica de concretos leves com argila expandida. Revista Materia, v. 15, n. 1, p. 031-039, 2010.

SCOBAR, R. L. Concreto leve estrutural: substituição do agregado graúdo convencional

\section{AGRADECIMENTOS}

Os autores agradecem à Concrecon pelo fornecimento da sílica ativa e do aditivo superplastificante utilizados neste trabalho. por argila expandida. 45p. Trabalho de Conclusão de Curso (Bacharelado) - Universidade Tecnológica Federal do Paraná. Campo Mourão, 2016.

SILVA, H. H. A. B. Caracterização mineralógica e filiação da vermiculita da Mina Cerrado III Sanclerlândia-GO. 2006. p. 1-127. Dissertação (Mestrado em Geologia) - Mineralogia e Petrologia, Universidade de Brasília, Brasília, 2006.

SOUSA, I. P. S. Obtenção de nanossílica funcionalizada visando o uso como aditivo em misturas cimentícias. 2017. 72 f. Dissertação (Mestrado em Química) - Universidade Federal de Goiás, Goniânia, 2017.

STOCCO, W.; RODRIGUES, D.; CASTRO, A.P.A.S. Concreto leve com uso de EPS. Cobenge 2009. n. 81, p. 1129-1134, 2009.

SUTCU, M. Influence of expanded vermiculite on physical properties and thermal conductivity of clay bricks. Ceramics International, v. 41, n. 2, p. 2819-2827, 2015.

TUTIKIAN, B. F.; HELENE, P. Dosagem dos Concretos de Cimento Portland. Concreto: Ciência e Tecnologia. São Paulo. Ibracon, 2011, cap 12.

UGARTE J. F. O, SAMPAIO J. A, FRANÇA S. C. A. Vermiculita. In: Rochas \& Minerais Industriais: Usos e Epecificações. Rio de Janeiro: CETEM, 2008. Cap 38. p.866-887.

VASKE, N. R.; CAMPAGNOLO, J. L.; DAL MOLIN, D. C. C. Relationship between the compressive strength of silica fume mortar applied to the substratum and the one obtained in standardized cylindrical test specimens. Revista IBRACON de Estruturas e Materiais, v. 3, n. 3, p. 322-345, 2010.

\section{AUTORES}

ORCID: https: / /orcid.org/0000-0001-6654-4586 MARINA COSTA DE SOUZA (MCS), Eng.| Centro Universitário Euro-Americano|Departamento de Engenharia Civil (UNIEURO) |Brasília, DF - Brasil | Correspondência para: SQN 307, BL I, AP 202 - Asa Norte, Brasília-DF, CEP: 70746-090 | e-mail: marinacosta015@gmail.com

ORCID: https://orcid.org/0000-0003-0102-762X AMÁLIA DE SOUSA DAVI, (ASD) Eng.| Centro Universitário Euro-Americano (UNIEURO) Departamento de Engenharia Civil | Brasília, DF Brasil | Correspondência para: QD 30, LT 22 - 
Jardim Brasília, Águas Lindas de Goiás-GO, CEP: 72915-111 | e-mail: amalia_davi@hotmail.com)

ORCID: https://orcid.org/0000-0002-0426-4615

YURI SOTERO BOMFIM FRAGA, (YSBF) M.SC. I Centro Universitário Euro-Americano (UNIEURO) I Departamento de Engenharia Civil | Brasília, DF . Brasil | Correspondência para: EQN 410/411, bloco A, apartamento 29 - Asa Norte, Brasília-DF, CEP: 70865-405 | e-mail: yurisotero.engcivil@gmail.com)

\section{HOW TO CITE THIS ARTICLE}

SOUZA, Marina Costa de; DAVI, Amália de Sousa; FRAGA, Yuri Sotero Bomfim. Efeito da Sílica Ativa nas Propriedades do Concreto Leve com Argila Expandida. MIX Sustentável, v. 8, n. 1, p. 29-40, jan. 2022. ISSN 24473073. Disponível em: <http://www.nexos.ufsc.br/index.php/mixsustenta vel> DOI: http://dx.doi.org/10.29183/24473073.MIX2022.v8.n1.29-40.

Submitted: $29 / 12 / 2020$

Approved: 25/05/2021

Published: 01/12/2021

Editora Responsável: Lisiane Itha Librelotto

Registro da contribuição de autoria:

Taxonomia CRediT (http://credit.niso.org/)

MCF, ASD, YSBF: análise formal, aquisição de financiamento, investigação, metodologia, visualização, escrita -rascunho original.

MCF, YSBF: conceituação, escrita - revisão e edição.

YSBF: administração de projetos, supervisão

Declaração de conflito: nada foi declarado. 